\title{
Difficulties in diagnosis of intracranial meningiomas by computed tomography
}

\author{
P PULLICINO, B E KENDALL, AND J JAKUBOWSKI \\ From the Lysholm Department of Neuroradiology, The National Hospital, London
}

SUMMARY The false positive and false negative computed tomography diagnoses of meningioma made using EMI 1010 and 5005 machines on a consecutive series of patients seen over a period of four years are described. About $1.2 \%$ of intracranial meningiomas were not detected on initial CT scan, $6.6 \%$ were misdiagnosed including $5.2 \%$ which were thought to be malignant lesions; in $9.4 \%$ of the cases identified as meningioma another diagnosis was subsequently established of which $6 \cdot 3 \%$ were malignant.

CT scanning shows an abnormality in patients with an intracranial tumour at the time of clinical presentation in a large majority of cases and earlier than any other radiological procedure. $^{1}$ This has modified the spectrum of preoperative investigation so that $\mathrm{CT}$ scanning has virtually replaced the combination of plain $\mathrm{X}$-rays, pneumography, angiography and isotope scanning that was previously necessary for diagnosis. A previous study ${ }^{2}$ has shown that the diagnosis of meningioma may be made on a precontrast scan in a high percentage of cases and this figure was further increased after postcontrast scanning. The experience gained from this study has been applied to routine examinations of scans and although complete reliance on CT has contributed to misdiagnosis in a few cases, the trend towards diminished use of other diagnostic methods for confirmation of the nature of the lesions has continued. We considered that analysis of the accuracy of CT in the diagnosis of intracranial meningiomas during this period would be of interest together with a critical appraisal of diagnostic errors.

\section{Methods}

The clinical and pathological record of all patients in the National Hospital for Nervous Diseases with a diagnosis of intracranial meningioma (excluding optic nerve sheath meningioma)

Address for reprint requests: Dr BE Kendall, The National Hospital Queen Square, London WC1N 3BG.

Accepted 14 April 1980 during the period of 1975 to 1978 were reviewed. All cranial CT scans in which a diagnosis of meningioma had been suggested during this period were also reviewed and data were correlated giving four groups:

(1) CT diagnosis correct.

(2) Meningioma present but no abnormality detected by $\mathrm{CT}$.

(3) Meningioma present but incorrectly diagnosed as a different pathology by CT.

(4) Another pathology incorrectly diagnosed as meningioma by $\mathrm{CT}$.

All examinations were performed on EMI 1010 or 5005 .

Enhanced scans were made after viewing the plain scans in over $95 \%$ of the cases. The method of enhancement was by intravenous injection immediately prior to scanning of $70 \mathrm{mls}$ sodium iothalamate containing $420 \mathrm{mgs} \mathrm{I} / \mathrm{ml}$.

\section{Results}

During the period of study a total of 347 patients with proven meningioma had CT examinations; $320(92.2 \%)$ were correctly diagnosed on the initial scan. The remaining $27(7.8 \%)$ were either considered to be normal (four cases), misdiagnosed as another pathology (19 cases) or diagnosed only as a non-specific tumour (four cases). The wrong diagnoses included glioma (13 cases), neurofibroma (two cases), metastases (one case), aneurysm (one case), craniopharyngioma (one case) and pituitary adenoma (one case). Twentysix of these cases had plain X-rays and 24 werc submitted to further studies. 
Angiography was performed in 22 of these 27 cases and in the majority (21) it showed a definite abnormality. However, the correct diagnosis was established in only 10 cases, in seven the findings were non-contributory, and in four the angiographic diagnosis was misleading (table 1). In the remaining 17 cases, misdiagnosed on CT scan, the correct diagnosis of meningioma was suggested by gamma scan (five cases), skull X-ray (two cases), encephalogram (two cases), and repeat CT scan (one case). A clinical diagnosis of meningioma was made in only five of these 27 cases. In 12 cases the correct diagnosis was not suggested prior to histological examination (table 2).
During the period under review meningioma was diagnosed on $353 \mathrm{CT}$ scans. Out of this number $84.9 \%(300)$ were confirmed by histology and $5.7 \% \quad(20)$ cases by angiography alone. Thirty-three cases $(9.5 \%)$ were proved to have a different pathology. The majority (22) of these $(67 \%)$ were malignant lesions. There were four cases of arterio-venous malformation and one aneurysm. The remainder were benign lesions including acoustic neuroma (two cases), pituitary adenoma (two cases) and craniopharyngioma (one case) (table 3). Thirty-one of these patients had plain X-rays and 25 were submitted to further study.

Angiography was performed on 25 cases

Table 1 Results of other investigations in meningiomas not detected or misdiagnosed on CT

\begin{tabular}{|c|c|c|c|c|c|}
\hline \multirow[t]{2}{*}{ Investigation } & \multirow{2}{*}{$\begin{array}{r}\text { Total } \\
\text { number }\end{array}$} & \multirow[t]{2}{*}{ Normal } & \multicolumn{3}{|l|}{ Positive } \\
\hline & & & Misleading & Non-Contributory & Diagnostic \\
\hline $\begin{array}{l}\text { Skull X-ray } \\
\text { Isotope scan } \\
\text { Angiogram } \\
\text { Pneumoencephalogram } \\
\text { Clinical }\end{array}$ & $\begin{array}{r}26 \\
11 \\
22 \\
7 \\
27\end{array}$ & $\begin{array}{l}12(46 \%) \\
1(9 \%) \\
1(4.5 \%) \\
1(14 \%) \\
0\end{array}$ & $\begin{array}{l}2(7 \cdot 7 \%) \\
0 \\
4(18 \%) \\
1(14 \%) \\
11(41 \%)\end{array}$ & $\begin{array}{r}10(39 \%) \\
5(45 \%) \\
7(32 \%) \\
3(43 \%) \\
11(41 \%)\end{array}$ & $\begin{array}{c}2(7.7 \%) \\
5(45 \%) \\
10(45 \%) \\
2(29 \%) \\
5(19 \%)\end{array}$ \\
\hline
\end{tabular}

Table 2 Methods of diagnosis of meningiomas not detected or misdiagnosed on CT. Diagnostic method suggesting correct diagnosis.

\begin{tabular}{|c|c|c|c|c|c|c|c|c|c|}
\hline CT Diagnosis & Total & $\begin{array}{l}\text { Angiogram } \\
(22)^{*}\end{array}$ & $\begin{array}{l}\text { Isotope } \\
\text { scan } \\
(I I)^{*}\end{array}$ & $\begin{array}{l}\text { Skull } \\
\text { radiograph } \\
(24)^{*}\end{array}$ & $\begin{array}{l}\text { Pneumoencephalo- } \\
\text { gram } \\
(7)^{*}\end{array}$ & Clinical & $\begin{array}{l}\text { Control } \\
C T \\
(3)^{*}\end{array}$ & $\begin{array}{l}\text { Total } \\
\text { cliagnosed } \\
\text { prior to } \\
\text { histology }\end{array}$ & $\begin{array}{l}\text { Histology } \\
\text { only }\end{array}$ \\
\hline Glioma & 13 & 3 & 3 & 0 & 1 & 1 & 0 & 7 & 6 \\
\hline Normal & 4 & 1 & $\mathbf{0}$ & 1 & 1 & 1 & 1 & 2 & 2 \\
\hline Non-specific tumour & 4 & 4 & 2 & 0 & 0 & 2 & 0 & 4 & 0 \\
\hline Neurofibroma & 2 & 0 & 0 & 0 & 0 & 0 & 0 & 0 & 2 \\
\hline Secondary tumour & 1 & 1 & 0 & 1 & 0 & 0 & 0 & 1 & 0 \\
\hline Craniopharyngioma & 1 & 0 & 0 & 0 & 0 & 0 & 0 & 0 & 1 \\
\hline Pituitary adenoma & 1 & 0 & 0 & 0 & 0 & 0 & 0 & 0 & 1 \\
\hline Aneurysm & 1 & 1 & 0 & 0 & 0 & 1 & 0 & 1 & 0 \\
\hline Totals & 27 & $10(45 \%)$ & $5(45 \%)$ & $2(8 \cdot 3 \%)$ & $2(23 \%)$ & $5(18.5 \%)$ & $1(33 \%)$ & $15(55 \%)$ & $12(45 \%)$ \\
\hline
\end{tabular}

* Total number of studies

Table 3 Method of diagnosis of lesions misdiagnosed as meningioma on CT. Diagnostic method suggesting correct diagnosis

\begin{tabular}{|c|c|c|c|c|c|c|c|c|}
\hline Final diagnosis & Total & $\begin{array}{l}\text { Angiogram } \\
(25)^{*}\end{array}$ & $\begin{array}{l}\text { Isotope } \\
\text { scan } \\
(14)^{*}\end{array}$ & $\begin{array}{l}\text { Skull } \\
\text { radiograph } \\
(31)^{*}\end{array}$ & $\begin{array}{l}\text { Pneumoencephalo- } \\
\text { gram } \\
(4)^{*}\end{array}$ & Clinical & $\begin{array}{l}\text { Total diagnosed } \\
\text { prior to } \\
\text { histology }\end{array}$ & $\begin{array}{l}\text { Histology } \\
\text { only }\end{array}$ \\
\hline Glioma & 13 & 3 & 1 & 1 & 0 & 2 & 5 & 8 \\
\hline Acoustic neurinoma & 2 & $\mathbf{0}$ & 0 & $\mathbf{0}$ & 0 & 1 & 1 & 1 \\
\hline Pituitary adenoma & 2 & 0 & 0 & 1 & 0 & 2 & 2 & 0 \\
\hline \multicolumn{9}{|l|}{ Arteriovenous } \\
\hline malformation & 4 & 2 & 0 & 0 & 0 & 0 & 2 & 2 \\
\hline Matastasis & 8 & 3 & 1 & $\mathbf{0}$ & 0 & 2 & 5 & 3 \\
\hline Craniopharyngioma & 1 & 0 & 0 & 0 & 0 & 0 & 0 & 1 \\
\hline Aneurysm & 1 & 0 & 0 & 0 & 0 & 0 & 0 & 1 \\
\hline Carcinoma (lachrymal) & 1 & 1 & 0 & 0 & 0 & 0 & 0 & 1 \\
\hline Granuloma & 1 & 1 & 0 & 0 & 0 & 0 & 1 & 0 \\
\hline Totals & 33 & $9(36 \%)$ & $2(14 \cdot 3 \%)$ & $2(6.5 \%)$ & 0 & $7(21 \%)$ & $16(48 \cdot 5 \%)$ & $17(51.5 \%)$ \\
\hline
\end{tabular}

- Total number of studies 
(76\%). All of the studies showed an obvious abnormality but in only nine cases was the angiogram diagnostic. In four cases it was noncontributory and in the remaining 12 it was misleading (table 4).

The initial CT misdiagnosis was corrected by other studies in $16(48.5 \%)$ of cases. In the remaining 17 patients $(51.5 \%)$ final pathology was only established by histological examination (table 3).

The scan findings in those meningiomas mistaken for other lesions and in the thirty-three cases which were mistaken for meningiomas are shown in table 5. In the malignant tumours in the latter group, moderate or marked oedema and especially oedema of the white matter occurred in $40 \%$ compared with about $10 \%$ in meningiomas in general; in those meningiomas mistakenly called gliomas or metastases on CT the incidence of moderate or marked oedema was only slightly higher (15\%). The incidence of mass effect and the pattern of enhancement was similar in both groups. In three cases a cystic meningioma was diagnosed as a glioma but cystic change was also present in histologically proven gliomas diagnosed as meningiomas. Two of the four arteriovenous malformations mistaken for meningiomas were thrombosed.

Table 4 Results of other investigations in lesions misdiagnosed as meningiomas on CT

\begin{tabular}{|c|c|c|c|c|c|}
\hline \multirow[t]{2}{*}{ Investigation } & \multirow{2}{*}{$\begin{array}{l}\text { Total } \\
\text { number }\end{array}$} & \multirow[t]{2}{*}{ Normal } & \multicolumn{3}{|l|}{ Positive } \\
\hline & & & Misleading & Non-contributory & Diagnostic \\
\hline $\begin{array}{l}\text { Skull X-ray } \\
\text { Isotope Scan } \\
\text { Angiogram } \\
\text { Pneumoencephalogram } \\
\text { Clinical }\end{array}$ & $\begin{array}{r}31 \\
14 \\
25 \\
4 \\
33\end{array}$ & $\begin{array}{l}14(45 \%) \\
1(7 \cdot 1 \%) \\
0 \\
0 \\
0\end{array}$ & $\begin{array}{c}3(9 \cdot 7 \%) \\
3(21 \cdot 4 \%) \\
12(48 \%) \\
3(75 \%) \\
18(55 \%)\end{array}$ & $\begin{array}{c}12(39 \%) \\
8(57 \%) \\
4(16 \%) \\
1(23 \%) \\
7(21 \%)\end{array}$ & $\begin{array}{l}2(6 \cdot 5 \%) \\
2(14 \cdot 3 \%) \\
9(36 \%) \\
0 \\
8(24 \%)\end{array}$ \\
\hline
\end{tabular}

Table 5

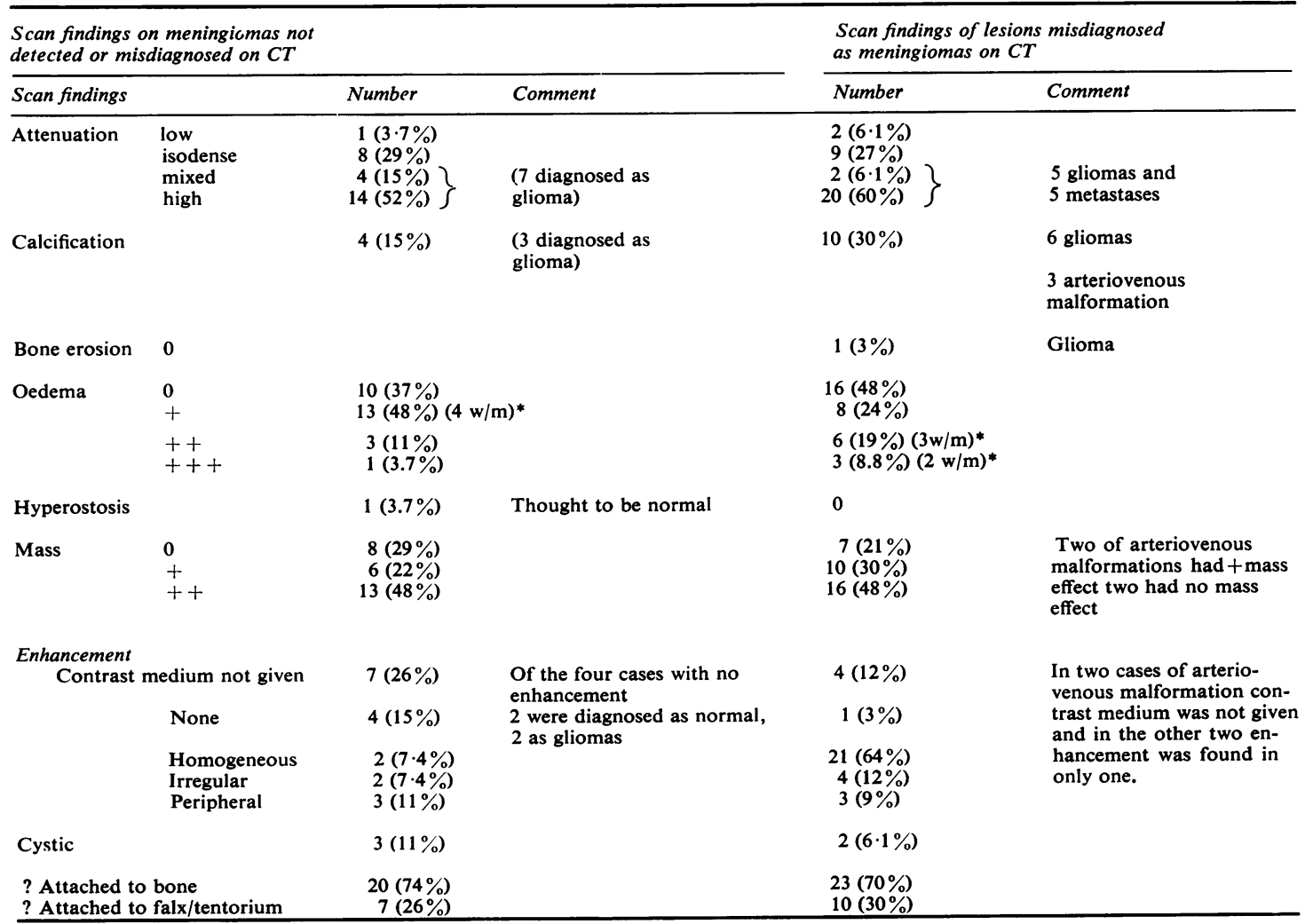

*Well defined low attenuation spreading through white matter. 
Table 6 Incident according to site

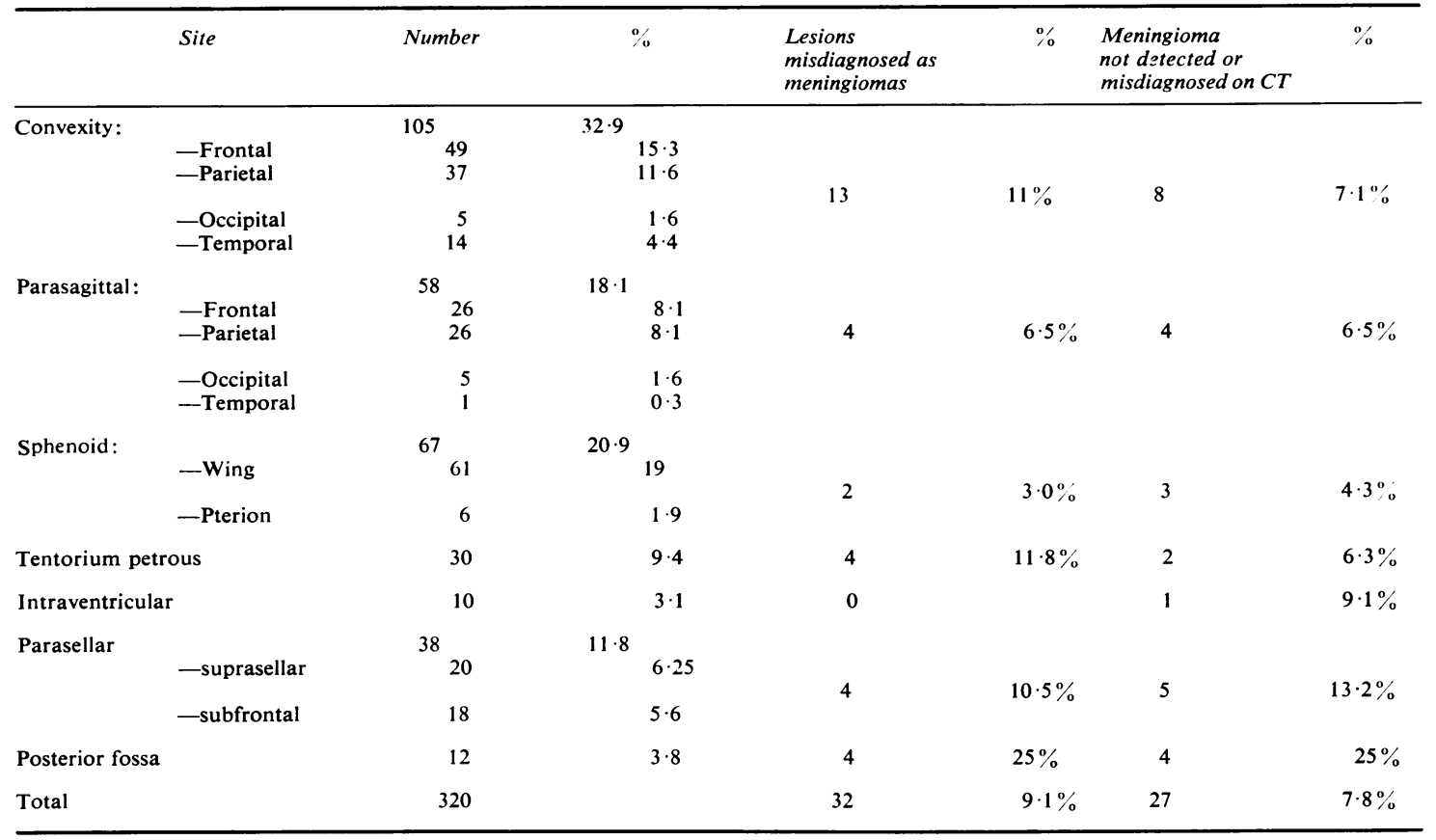

Three of the arteriovenous malformations were partly calcified lesions and only one of the two given contrast medium showed enhancement.

Table 6 shows the distribution of the meningiomas in this series together with the site of incorrectly diagnosed lesions. In general agreement with other series ${ }^{3}$ sphenoid ridge or wing meningiomas are most common, followed by frontal and parietal convexity tumours. The proportion of incorrect diagnoses is similar in all regions except for the posterior fossa, where there is a much higher incidence of both false positive and negative misdiagnosis, and the spenoidal ridge where, largely because of the high incidence of bone sclerosis misdiagnosis is less frequent.

\section{Discussion}

The detection rate of almost $99 \%$ confirms that CT is the most accurate screening test for meningioma. The undetected tumours were one en plaque cerebellar convexity tumour diagnosed by skull X-ray; one suprasellar tumour diagnosed only at surgery, causing compression of an optic nerve; one parasellar lesion shown by air encephalogram and follow-up CT; one parasagittalfalx meningioma, diagnosed by follow-up CT. The last three tumours were not shown even in retrospect on the initial $\mathrm{CT}$. In one case, the parasagittal tumour, contrast medium was not given for the first study.

The typical sites of meningiomas and well demarcated edges suggesting their extracerebral origin, especially when combined with their characteristic attenuation higher than that of the adjacent brain sometimes with psammomatous calcification and usually with homogeneous enhancement and little adjacent cerebral oedema in the white matter suggest the diagnosis, which may be confirmed by observing hyperostosis $(16 \%)$ at the site of attachment to bone. Less frequently the bone is shown to be destroyed from the inner table $(3 \%)$ and this also suggests the diagnosis in tumours adjacent to the vault. The meningiomas which were misdiagnosed as glioma were either intraventricular (one) (fig 1), irregularly lobulated cavitating masses (four) (fig 2) relatively small in relation to extensive white matter oedema (two) or the extracerebral attachment was not appreciated (six) (fig 3). Throughout the study it was realised that such atypical CT features could occur with meningiomas and led to four of them being diagnosed merely as peripheral intracranial tumour with the implication that further study was necessary to determine their type. Some of the difficulties 

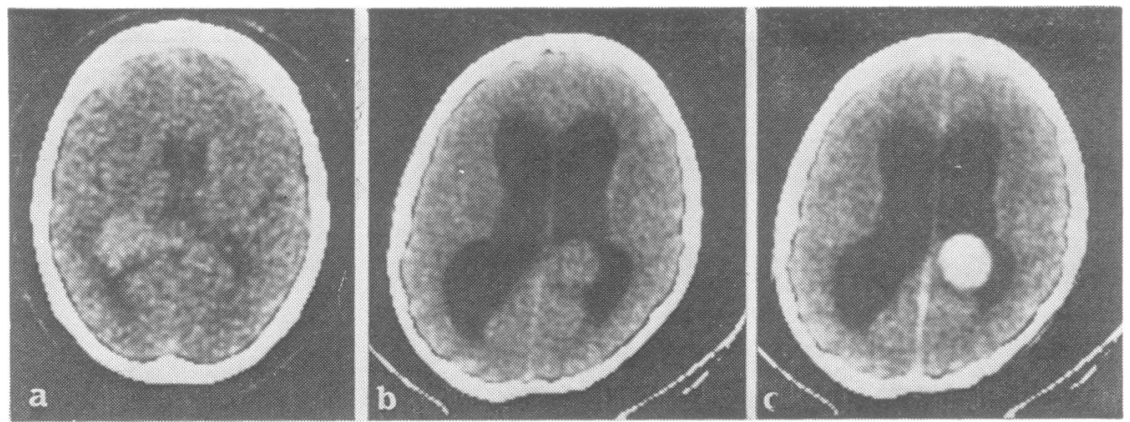

Fig 1 (a) Intraventricular meningioma. Plain scan. Irregular region of raised attenuation in left trigone region. Thought to be intracerebal and diagnosed as a glioma. (b), (c) Deep right parietal II astrocytoma. (b) Plain scan, (c) after contrast medium. The lesion appears to be intraventricular and shows dense enhancement. Diagnosed as intraventricular meningioma.
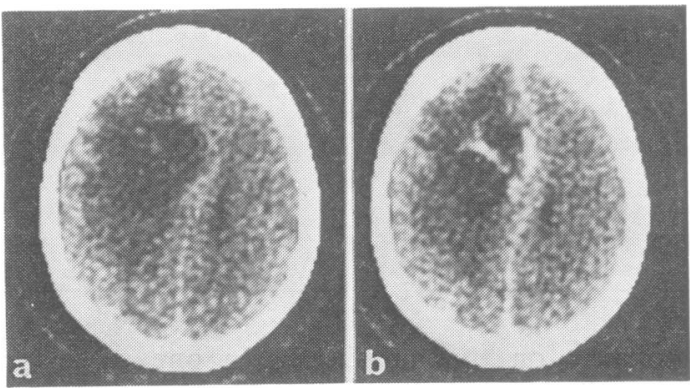

Fig 2 Meningioma. (a) Plain scan, (b) after contrast medium. The tumour is of low attenuation and has moderate surrounding oedema. There is irregular peripheral enhancement.
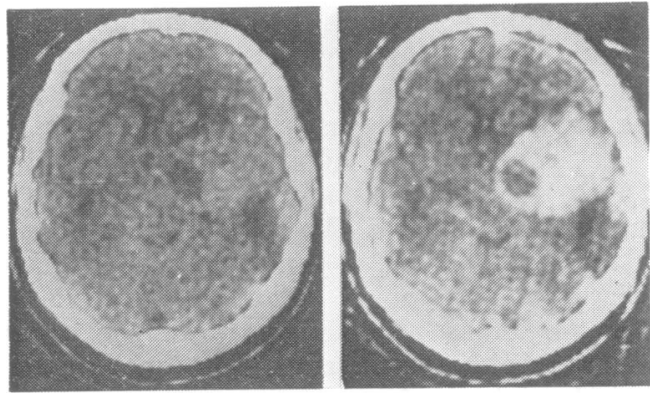

Fig 3 Meningioma. (a) Plain scan, (b) after contrast medium. Irregular mass of slightly increased attenuation showing dense enhancement with little peripheral oedema. Extracerebral attachment not appreciated.

are due to tumours being attached along the high and low convexities; coronal sections or coronal and sagittal reconstructions should be helpful in diminishing the number of errors in these situations. Movement artefact was prominent in one case and contrast enhancement was omitted in five.

Most gliomas are of mixed attenuation, close to and below that of brain. Under $20 \%$ are of increased attenuation and show homogeneous enhancement. However, over $50 \%$ of metastases are of increased attenuation and two-thirds show homogeneous enhancement. The combination of a solitary peripheral malignant tumour with such characteristics is much less frequent but accounted for two-thirds of the false positive diagnoses of meningioma (fig 4). Our analysis shows that the incidence of moderate or marked intracerebral oedema (fig 5) is greater in these tumours and in some cases the tumour margin was rather ill defined (fig 6), which is uncommon with meningiomas. Two microgliomas were included in the group; they exactly simulated meningiomas and had little surounding oedema. These tumours usually occur deep in the cerebral substance close to the central structures. One primary cerebral lymphoreticular tumour was also included. It involved the temporal and inferior frontal lobes with bilateral white matter oedema. In retrospect the septum pellicidum is obviously thickened (fig 7). Misdiagnosis is more significant when a benign pathology is mistaken for a glioma than vice versa. Hence there is a tendency to suggest more strongly the possibility since such a suggestion will invariably lead to biopsy and often to angiographic examination.

The difficulty with differential diagnosis of cerebello-pontine angle masses and with perisellar lesions is well known. ${ }^{4}$ A small percentage of neurofibromas, craniopharyngiomas and pituitary adenomas have CT characteristics more commonly found with meningiomas and vice 


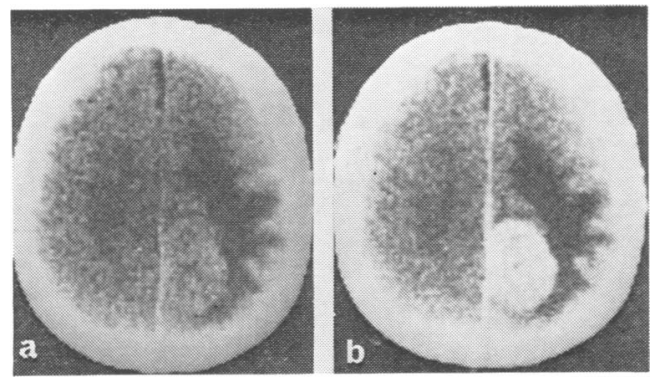

Fig 4 Secondary carinoma. (a) Plain scan (b) after contrast medium. Mass of slightly raised attenuation with surrounding oedema, and dense enhancement. Diagnosed as meningioma.

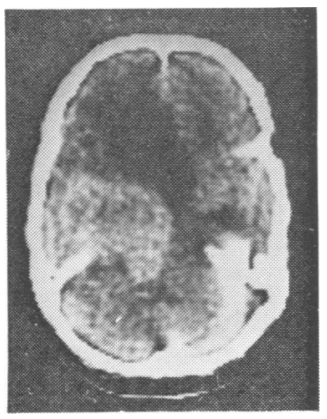

Fig 5 Glioblastoma. Plain scan. Raised attenuation well defined temporal lobe lesion extending through tentorium with extensive oedema anterior to it. Thought to be a meningioma arising from the left petrous ridge.
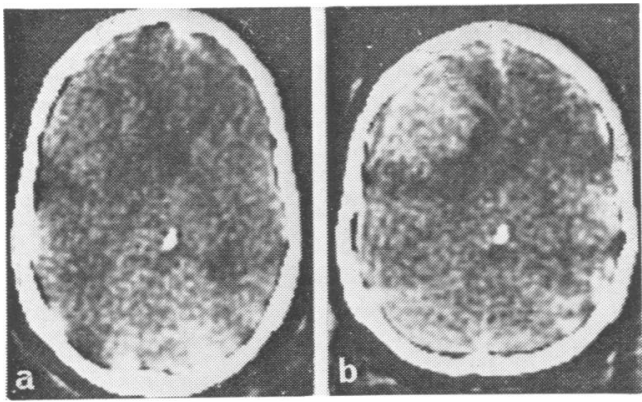

Fig 6 Oligodendroglioma. (a) Plain scan (b) after contrast medium. Peripherally placed mass of increased attenuation with low attenuation medially thought to be a capping cyst. Diagnosed as meningioma.

versa (fig 8). Bone changes shown on plain X-ray and, occasionally, angiography are helpful in suggesting the correct diagnosis. Large, partly thrombosed aneurysms are of high attenuation, sometimes with peripheral calcification and diagnosis is confirmed when there is dense central enhancement. The clearly demarcated slightly increased attenuation and marked total enhancement of a patent aneurysm, is also generally appreciated (fig 9) and the diagnosis at least sufficiently suspected to lead on to angiography.

of particular interest are the four angiomatous malformations (AVM). Failure to appreciate this diagnosis pre-operatively is potentially hazardous. They are of increased attenuation due to content of blood or thrombus and frequently calcification in the walls of the blood vessels or damaged brain. There is also often microcyst formation or local atrophy causing regions of low attenuation and the enlarged blood vessels frequently show as round, oval or serpiginous aggregations depending on their orientation to the CT section, especially when they are enhanced by intravenous contrast medium. The enhancement is partly within the patent blood vessels but is also due to alterations in the blood brain barrier so that it is usually present even when the malformations are thrombosed. ${ }^{5}$ Mass effect occurs in less than $20 \%$ of angiomas unless there has been haemorrhage ${ }^{6}$; it is not usually marked and there is generally little or no low attenuation in the white matter beyond the confines of the AVM. All our AVMs mistaken for meningiomas (fig 10) were peripheral high attenuation lesions; three were partially calcified, two had slight mass effect, but none had any surrounding low attenuation. The two completely thrombosed malformations were rescanned with contrast medium and one enhanced. They were diagnosed only at operation but two others were patent and shown at angiography. CT features which in retrospect could have suggested the correct diagnosis were present in two and in none was there supporting evidence for the CT diagnosis of meningioma from any other study.

Two other lesions that may possibly be mistaken for meningioma are:

(1) A large plaque of ossification projecting from the dura. However the absence of any adjacent unossified mass, of any abnormal enhancement and of any changes in the skull should suggest the correct interpretation.

(2) An organising intracerebral haematoma extending superficially may rarely simulate a meningioma when the lesion remains of greater than brain density and enhancement is more extensive than the usual peripheral ring. Even then $C T$ appearance will generally be atypical for meningioma and a detailed history will almost always be helpful by suggesting a resolving acute lesion.

An atypical irregular enhancement pattern was one cause of a mistaken diagnosis in mengioma but total absence of enhancement occurred 

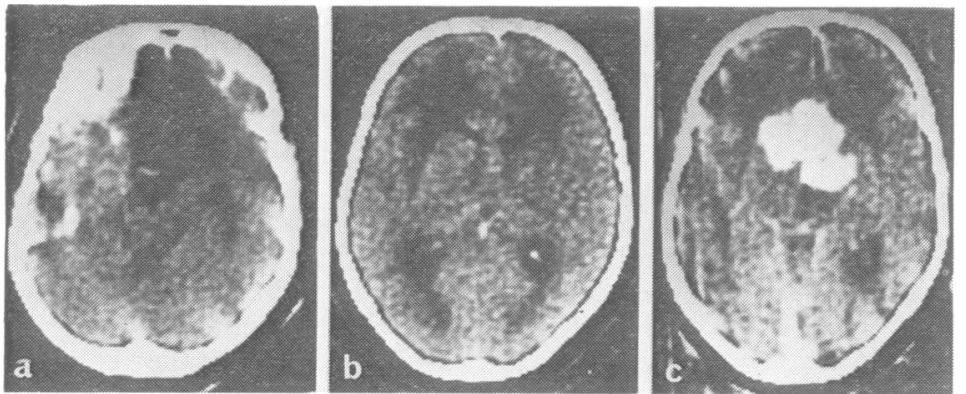

Fig 7 Lymphoreticular tumour temporal and inferior frontal extending into septum pellucidum. $(a, b)$ Plain scan (c) after contrast medium, mixed high attenuation and isodense mass with surrounding oedema. Marked enhancement after conray. Thought to be a meningioma.
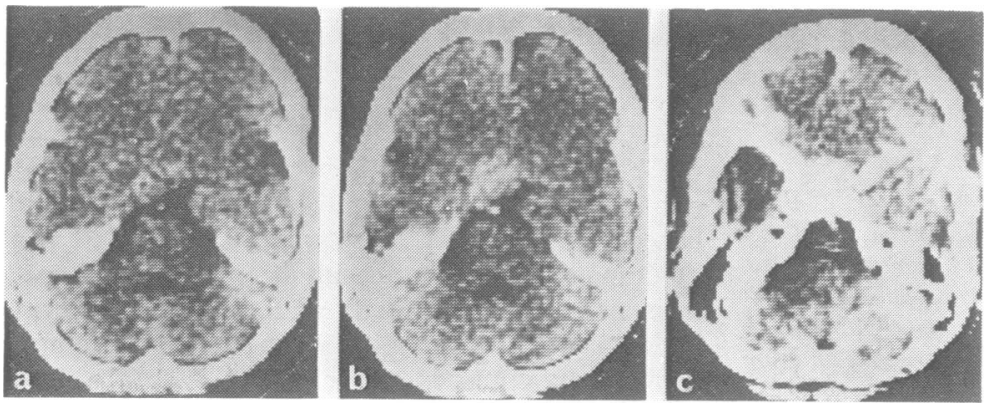

Fig 8 Meningioma. (a) Plain scan $(b, c)$ after contrast medium. Isodense mass showing enhancement in and around the sella. Diagnosed as pituitary adenoma.
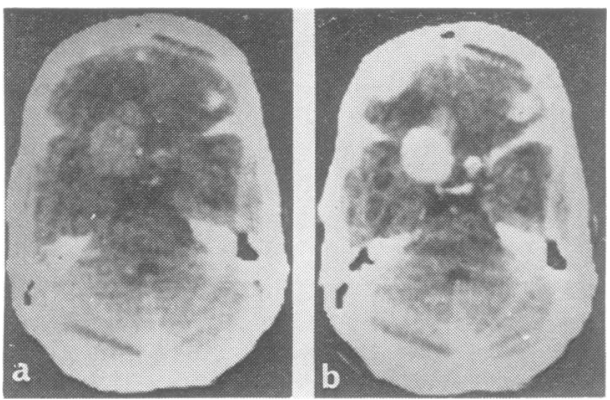

Fig 9 Meningioma. (a) Plain scan (b) after contrast medium. Rounded high attenuation suprasellar mass with dense enhancement. Diagnosed as aneurysm.
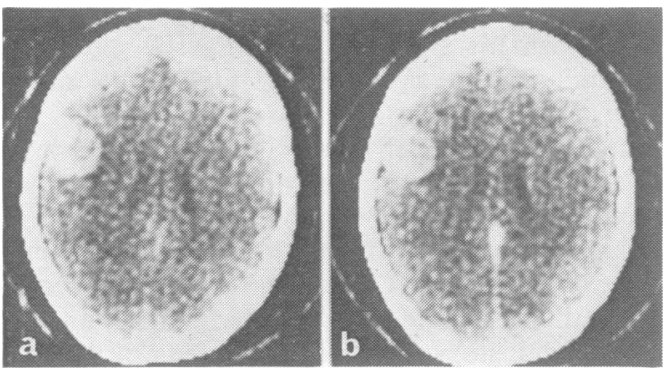

Fig 10 Thrombosed arteriovenous malformation. (a) Plain scan (b) after contrast medium. Peripheral mass of raised attenuation showing some enhancement with conray. Diagnosed as meningioma. in only two cases; conversely however the homogeneous and dense nature of enhancement of some lesions was a contributory factor in their being misdiagnosed as meningiomas. It is therefore unlikely that larger amounts of iodine given by bolus or contrast infusion during the examination would have favourably influenced our diagnostic accuracy.

Plain X-rays show bone changes related to the attachment of the meningioma, prominent vascular grooves leading to this region or calcification within the tumour in some $30-50 \%$ of cases $^{7}$ and it has been claimed that a specific diagnosis of meningioma is possible by angiography in over $70 \%$ of cases. ${ }^{8} 9$ However, in our unusual cases in which CT diagnosis was difficult the plain X-rays were diagnostic in only $12.5 \%$ and, though angiography revealed a mass in all except one case, it was diagnostic of meningioma in only $45 \%$ and in $18 \%$ the angiographic report suggested a malignant tumour. Similarly, in the cases misdiagnosed as meningioma on CT scan the angiogram was considered to support this diagnosis in almost $50 \%$. Only eight (24\%) of lesions misdiagnosed as meningioma, and three $(11 \%)$ of the meningiomas undetected or misdiagnosed on CT had no other studies than CT and plain X-rays, the remainder having angiography, isotope scan or pneumoencephalography.

It may be considered unsatisfactory that, despite being aware of the difficulties of CT 
diagnosis, there was still a false negative rate of $7.8 \%$ and a false positive rate of $9.5 \%$, but CT is the most accurate method for detection and specific diagnosis of meningioma. Other radiological methods are also particularly accurate in the diagnosis of this tumour, but are less valuable in the difficult cases which have not been diagnosed by computed tomography.

Despite computed tomography and other radiological procedures an incorrect diagnosis was rectified only by biopsy in $3.2 \%$ of meningiomas and $4.8 \%$ of the lesions diagnosed as meningiomas were shown on histology to be other conditions, mainly malignant tumours.

\section{References}

1 Wende S, Aulich A, Schindler E et al. A German multicentre study of intracranial tumours. In: The First European seminar on computed axial tomography in clinical practice, DuBoulay GH, Moseley IF, eds. Berlin: Springer Verlag, 1977: 111-7.

2 Sutton D, Claveria LE. Meningiomas diagnosed by scanning: A review of 100 intracranial cases. In: The first European seminar on computerised axial tomography in clinical practice, DuBoulay GH, Moseley IF, eds. Berlin: Springer Verlag 1977: 102-10.

3 Logue, Valentine. Surgery of meningiomas. In: Operative surgery, Neurosurgery, 3rd edition, Lindsay Symon, ed. London and Boston: Butterworths, 1979: 128.

4 Möller A, Hatam A, Olivecrona N. The differential diagnosis of pontine angle meningioma and acoustic neuroma with computed tomography. Neuroradiology 1978; 17, 1:21-3.

5 Bell BA, Kendall BE, Symon L. Angiographically occult arteriovenous malformations of the brain. J Neurol Neurosurg Psychiat 1978; 41:1057-64.

6 Kendall BE, Claveria LE. Use of computerised axial tomography for the diagnosis and management of intracranial angiomas. Neuroradiology 1976; 12:141-60.

7 Traub SP. Roentgenology of intracranial meningiomas. Springfield: Charles C Thomas 1961: 88

8 Jacobson HE, Lubetzky HW, Shapiro JE, Carton C. Intracranial meningiomas: a Roentgen study of 126 cases. Radiology 1959; 72:356-67.

9 Wickbom I, Stattin S. Roentgen examination of intracranial meningiomas. Acta Radiol (Stockh) 1958; 50:175-82. 